\title{
A Spectrum Handoff Scheme by Considering the Interference Probability in Cognitive Radio
}

\author{
Hong Du, Shuang Fu and Tingyi Shang \\ College of information technology, Heilongjiang Bayi Agricultural University, \\ Daqing and 163319, China \\ duhong929@163.com,fushuang_dq@163.com
}

\begin{abstract}
In cognitive radio networks, spectrum sensing is used to detect the spectral state of authorized users. In particular, upon detecting primary users (PUs) appearances, Secondary User (SU) vacates the channel and performs the spectrum handoff. In order to protect the PUs, interference constrains and reserved channel are considered. If the $S U$ can't find any idle channel after the whole spectrum sensing, it carries out spectrum handoff to the reserved channel. In addition, spectrum handoff delay is derived. The nonlinear constrained optimization problem for the presented approach is formulated. In special, the goal is to minimize the SU's handoff delay while protecting the PU from the interference. Numerical results discuss the impact factors on the handoff delay. Lower interference constrains can not only protect the PUs, but also can provide the lower handoff delay for SUs.
\end{abstract}

Keywords: cognitive radio, spectrum handoff, handoff delay, interference probability, spectrum sensing

\section{Introduction}

Recently, cognitive Radio (CR) has been proposed to effectively utilize the spectrum due to the spectrum underutilization of current spectrum allocation policy [1]. CR refers to that wireless systems are capable of sensing and reconfiguration. In the same frequency range, there are two co-existing systems: primary system and secondary system. Primary system refers to the licensed system with legacy spectrum. This system has the exclusive privilege to access the assigned spectrum. Secondary system refers to the unlicensed cognitive system and can only opportunistically access the spectrum holes which are not used by the primary system. We call the subscriber in the primary system as Primary User (PU) and the subscriber in the secondary system as Secondary User (SU). The SUs are able to dynamically access the licensed frequency bands without any modification to the primary system.

Although the motivation of CR technology is simple, the design and implementation have a large number of challenges. Spectrum mobility or called spectrum handoff issue, which is discussed less often in the literature than other spectrum issues. There are two phases in spectrum handoff: PU detection and link maintenance. Once a PU was detected to be appearance, the SU has to vacate the channel for the PU. After the channel release, the SU will perform the link maintenance to re-construct the communications in order to avoid service termination. During this procedure, the SU may search the spectral band and transfer its communications to another available spectrum, if available. This procedure is referred as spectrum handoff.

Compared to spectrum sensing, there are few studies on spectrum handoff issue. For instance, Channel Switching policies and a proactive spectrum handoff protocol are proposed to let unlicensed users vacate a channel before a licensed user utilizes it to 
avoid unwanted interference [2]. Son, et. al., incorporate proactive spectrum sensing into the proposed spectrum handoff scheme to reduce the number of spectrum handoff [3]. In addition, author relies on multi-agent negotiation to enable cognitive radio terminals switching towards the best available spectrum band [4]. An analytical framework is presented to assess the impact of common control interface incorporation into CR nodes for spectrum handoff [5]. Besides, Wu et.al propose a spectrum handoff scheme based on Recommended Channel Sensing Sequence, which aims to optimize the spectrum handoff delay subject to the sensing reliability and link maintenance constraints [6]. In special, Wang et.al present an analytical framework to evaluate the effects of multiple spectrum handoffs on channel utilization and latency performance [7]. From a different perspective, Coutinho proposes a new spectrum handoff scheme that considers the existence of errors in primary user detection to achieve a better channel ordering in terms of spectrum utilization efficiency and primary user interference [8]. It is observed that, in these studies, the QoS provision of SUs and protection of PU in spectrum handoff scheme has not been discussed.

In this paper, we present three aspects different from the existed studies. Firstly, two channels are discussed from existing studies on spectrum handoff, from a more realistic environment perspective, we consider multiple channels situation for the spectrum handoff. Secondly, we consider the impact of spectrum sensing on spectrum handoff. Besides, we take into account the interference probability constrains for protecting PU and set a reserved channel for providing the QoS of SU in spectrum handoff. Finally, average handoff delay was deduced to characterize spectrum handoff performance.

The rest of the paper is organized as follows. In Section 2, we introduce the system model of spectrum handoff. In Section 3, we describe the proposed spectrum handoff scheme for three stages. In Section 4, we investigate the performance optimization and deduce the average handoff delay. In Section 5, we provide the simulation results and discussion. Finally, Section 6 concludes this paper.

\section{System Model}

In cognitive radio networks, spectrum handoff occurs when the high-priority PU appears at its licensed channel and the channel is occupied by SUs. In this case, SUs are forced to vacate the occupied licensed spectrum. Spectrum handoff procedures aim to help SUs to find suitable target channels to resume the unfinished transmission.

In general, there are two major types of spectrum handoff schemes. One is the reactive-sensing spectrum handoff, where the target channel for spectrum handoff is sensed only after the spectrum handoff request is made. In this case, the instantaneous outcomes from spectrum sensing will be used to determine the target channel selection for spectrum handoff. The other one is the proactive-sensing spectrum handoff, for which the target channel is predetermined. In this case, SUs periodically observe all channels to obtain the channel usage statistics, and determine the candidate set of target channels for spectrum handoff according to the long-term observation outcomes.

The advantage of reactive-sensing spectrum handoff is the accuracy of the selected target channel, but pays the cost of sensing time. By contrast, the proactive-sensing spectrum handoff avoids the sensing time, but the predetermined target channel may not be available. Intuitively, the proactive-sensing spectrum handoff maybe has shorter data transfer time because at the moment of link transition it does not need to scan a huge spectrum to determine the target channel. On the other hand, the reactive-decision spectrum handoff maybe has longer data transfer time because spectrum sensing technology can help the SUs find the idle channels at the instant that spectrum handoff request is made.

From a realistic perspective, we investigate a spectrum handoff scheme for multiple channels situation. Figure 1 shows the spectrum handoff process. We assumed that the 
spectrum handoff of SUs in multiple channels environment. Considering the QoS provisioning of SUs, we set the reserved channel.

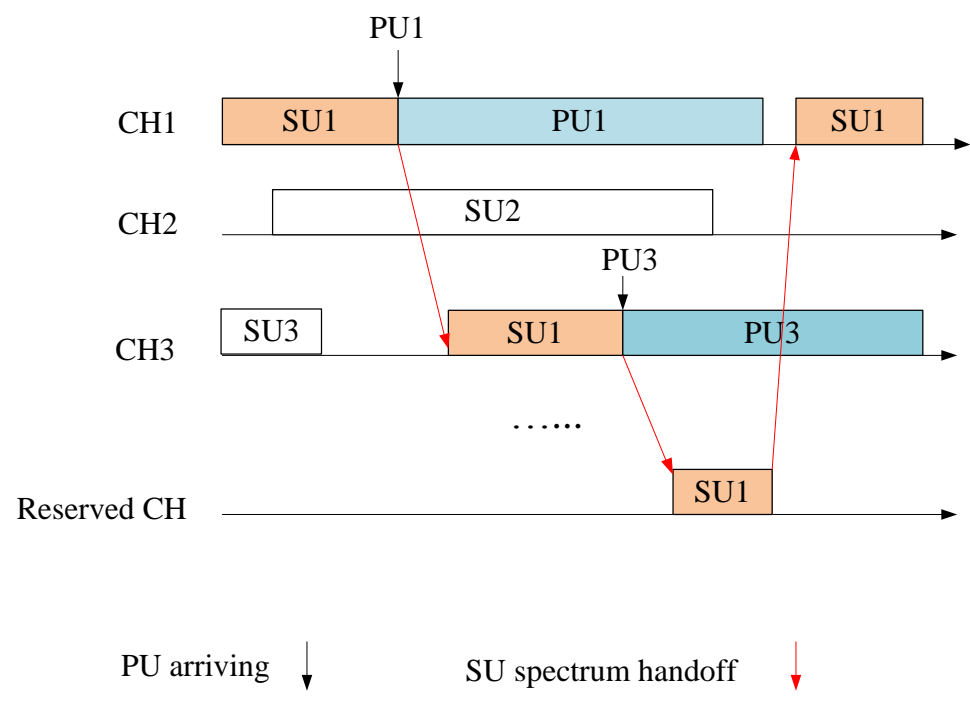

\section{Figure 1. The Spectrum Handoff with Reserved Channel}

The spectrum handoff scheme is described as follows. We assumed that SU1 occupied the channel $1(\mathrm{CH} 1)$ firstly, upon detecting a PU, SU1 vacates its channel to the PU, then it carries out spectrum sensing to the other channel in sequence before spectrum handoff. As shown in Fig.1, SU1 senses $\mathrm{CH} 2$ is occupied and $\mathrm{CH} 3$ is idle, if the collision probability from SU is less than the collision constrains threshold of PU, it performs spectrum handoff to CH3. Upon SU1 detecting PU3 arrive, it senses the other channel, if it can't find any idle channel; it carries out spectrum handoff into the reserved channel. Then it keeps on performing spectrum sensing periodically, if SU1 finds $\mathrm{CH} 1$ is idle, it vacates the reserved channel and performs spectrum handoff to $\mathrm{CH} 1$.

\section{The Proposed Spectrum Handoff Scheme}

For the proposed spectrum handoff scheme, we divided it into three stages: PU's arriving stage, SU's spectrum sensing stage and SU's spectrum handoff stage, respectively. Therefore, we will specify the three stages in detail as follows.

\subsection{PU's Arrival Model}

A primary network with the model is discussed as follows. This established prediction on the semi-Markov model is approximated by Continuous-time Markov chain with a good fitting exponential distribution as idle and busy periods [9]. Therefore, we follow the same model with exponential parameters $\alpha$ and $\beta$ for idle and busy state, whose probability density functions are expressed in the following.

$$
\begin{aligned}
& f_{\text {idle }}(t)=\alpha \exp (-\alpha t) \\
& f_{\text {busy }}(t)=\beta \exp (-\beta t)
\end{aligned}
$$

Therefore, the stationary distributions of the idle and busy period are expressed in the following. $P\left(H_{0}\right)$ and $P\left(H_{l}\right)$ denote the probability of idle and occupied channel by PUs. 


$$
\begin{aligned}
& P\left(H_{0}\right)=\frac{\beta}{\alpha+\beta} \\
& P\left(H_{1}\right)=\frac{\alpha}{\alpha+\beta}
\end{aligned}
$$

\subsection{SU's Spectrum Sensing}

In cognitive radio networks, SUs sense the spectrum of the PUs and opportunistically utilize any spectrum holes within the PU bands. While doing so, SUs are required not cause any undue interference to active PUs. Consequently, in order to avoid the collision to PUs, SUs need to sense the spectrum periodically to ensure the absences of PUs.

$P_{d}$ and $P_{f}$ are two important parameters for spectrum sensing, where $P_{d}$ is the probability of detection and $P_{f}$ is the probability of false alarm, respectively. $P_{d}$ is the probability that SUs must accurately detect the presence of active PUs. The higher value of $P_{d}$, the better protection for PUs. According to one example set up in [10], the required probability of detection is above $90 \%$ for cognitive radio systems operating on VHF/UHF TV bands. $P_{f}$ is the probability that SUs falsely detect the presence of PUs when in fact none of them is active. From the viewpoint of SUs, the lower the value of $P_{f}$, the higher the spectrum utilization.

Let Ts be the duration that each SU carries out spectrum sensing, when $P_{d}$ and signal to noise ratio (SNR) $\gamma$ are fixed, given a sensing algorithm, $P_{f}$ is a non-increasing function of Ts [11].

We assumes that the primary signal is independent and identically distributed with zero mean; the noise at SU is circular symmetric complex Gaussian with zero mean; and the primary signal and noise are independent. Hence, based on energy detection algorithm, the probability of false alarm is calculated by:

$$
P_{f}\left(T_{s}\right)=\left(\sqrt{2 \gamma+1} Q^{-1}\left(P_{d}\right)+\sqrt{T_{s} f_{s}} \gamma\right)
$$

Where $f_{s}$ is the channel sampling rate and $\mathrm{Q}($.) is the complementary distribution function of a standard Gaussian variable, i.e.,

$$
Q(x)=\frac{1}{\sqrt{2 \pi}} \int_{x}^{\infty} e^{-t^{2} / 2 d t}
$$

\subsection{SU's Spectrum Handoff}

In the following, we will present the spectrum handoff procedure. We assumed that the spectrum handoff scheme for the SUs is studied in multiple channels environment. Considering the interference avoiding to PUs and QoS provisioning of SUs, we set the interference probability threshold of PUs and reserved channel for SUs. The spectrum handoff scheme is described in detail as follows.

Step 1: SU occupies the $\mathrm{CH} 1$ which is idle and performs the spectrum sensing periodically.

Step 2: Upon detecting a PU's arriving, SU carries out spectrum sensing to the other channels in sequence.

Step 3: If interference probability is less than interference threshold of PU, it performs spectrum handoff to the idle channel.

Step 4: Upon SU detecting PU's arriving, it senses the other channels, if it can't find idle channel after spectrum sensing for all the channels, it carries out spectrum handoff 
into the reserved channel.

Step 5: Then SU performs spectrum sensing periodically, if SU finds $\mathrm{CH} 1$ is idle, it vacates the reserved channel and performs spectrum handoff to $\mathrm{CH} 1$.

\section{Performance Optimization of Spectrum Handoff}

In this section, the spectrum handoff delay in the proposed scheme is presented and derived in detail. Finally, we present the problem optimization of spectrum handoff approach.

\subsection{Analysis of Handoff Delay}

In order to protect the PU from interference, SU vacates the channel upon a PU's appearance and performs the spectrum sensing. Here we consider the imperfect spectrum sensing impact on the spectrum handoff. On the one hand, the PU reclaims the channel in fact, and the SU detects the PU correctly; On the other hand, the SU detects the PU while the PU doesn't return to the channel in fact. Therefore, the probability that SU vacates the channel $P_{v}$ is expressed as follows.

$$
\mathrm{P}_{v}=P_{d} P\left(H_{1}\right)+P_{f} P\left(H_{0}\right)
$$

After SU vacates the channel, it performs the spectrum sensing to find the available channel. There are two cases for the spectrum handoff: on the one hand, there exists the available channel after spectrum sensing in sequence. On the other hand, there is no available channel. The probability of two cases mentioned above is denoted as follows,

$$
\begin{gathered}
P_{\text {available }}=1-P\left(H_{1}\right)^{N} \\
P_{n o \_a v a i l a b l e}=P\left(H_{1}\right)^{N}
\end{gathered}
$$

where $N$ is the number of channels.

The handoff delay is the key metric for the QoS provision of SU in spectrum handoff. In specific, handoff delay is defined as the duration from the instant of pausing data transmission until the instant of resuming the transmission of SU.

In the following, we will discuss the handoff delay based on two cases as follows.

\subsubsection{Case 1}

If there exists the available channel, that means SU could find the idle channel for its handoff after some times spectrum sensing. In special, if SU senses the $\mathrm{CH} 2$ is idle, the handoff delay is the consumed sensing time $T s$. If SU senses the $\mathrm{CH} 2$ is busy and $\mathrm{CH} 3$ is idle, the handoff delay is the double sensing time $2 T s$. Similarly, If SU senses the final $\mathrm{CH}$ is idle, the handoff delay is $(N-1) T s$.

\subsubsection{Case 2}

Once there is no available channel after spectrum sensing for all the channels, that means all the channels are in busy state, then the SU performs spectrum handoff to the reserved channel after $N$ times sensing. Therefore, the handoff delay in this case is ( $N$ 1)Ts.

Therefore, once the SU detects the PU's arriving whether it is correct detection or false alarm, SU still needs to vacate the channel to the PU, and performs the spectrum sensing to the other channels. Based on two cases analysis of handoff mentioned above, the handoff delay is derived as follow in detail. 


$$
\begin{aligned}
\mathrm{T}_{\text {delay }}= & P_{v} \cdot\left\{P _ { \text { available } } \cdot \left[P\left(H_{0}\right) \cdot T s+P\left(H_{1}\right) P\left(H_{0}\right) \cdot 2 T s\right.\right. \\
& \left.\left.+\ldots+P\left(H_{1}\right)^{N-1} P\left(H_{0}\right) \cdot(N-1) T s\right]+\left[P_{n o \_} \text {available } \cdot(N-1) T s\right]\right\}
\end{aligned}
$$

Combining (7), (8) and (9), we have the handoff delay for $\mathrm{N}$ channels as follows:

$$
\begin{aligned}
\mathrm{T}_{\text {delay }}=[ & \left.P_{d} P\left(H_{1}\right)+P_{f} P\left(H_{0}\right)\right] \\
& \cdot\left\{[ 1 - P ( H _ { 1 } ) ^ { N } ] \cdot \left[P\left(H_{0}\right) \cdot T s+P\left(H_{1}\right) P\left(H_{0}\right) \cdot 2 T s\right.\right. \\
& \left.\left.+\ldots+P\left(H_{1}\right)^{N-1} P\left(H_{0}\right) \cdot(N-1) T s\right]+\left[P\left(H_{1}\right)^{N} \cdot(N-1) T s\right]\right\}
\end{aligned}
$$

\subsection{Problem Optimization of Proposed Spectrum Handoff}

The aim of spectrum handoff is to protect PUs avoiding the interference, and ensure that the QoS provisioning of SUs. Therefore, we investigate the spectrum handoff not only focus on spectrum handoff performance but also the interference to PUs and QoS provisioning of SUs.

We further assumed that $P_{d}$ is equal to the required value $P_{d}{ }^{*}$, $\mathrm{Pf}$ is no more than a maximum value $P_{f}^{*}$. The handoff delay optimization based on interference constrained is expressed as follows:

$$
\begin{array}{ll}
\min & \mathrm{E}\left[\mathrm{T}_{\text {delay }}\right] \\
\text { s.t. } & \mathrm{P}_{\mathrm{i}}<\zeta \\
& \mathrm{P}_{\mathrm{d}}=P_{d}^{*}, P_{f} \leq P_{f}^{*}
\end{array}
$$

Where $\zeta$ is the interference constraint for the PU.

Therefore, we will investigate the probability of interference to the PU due to imperfect spectrum sensing.

To give a good protection, it is required that the interference to PUs should be limited by a certain target value. When PU is active but SU cannot detect PU correctly, the probability of interference $P_{i}$ is expressed by

$$
\mathrm{P}_{\mathrm{i}}=P\left(H_{1}\right) \cdot\left(1-P_{d}\right) \cdot \frac{T_{P}-T s}{T_{P}}=\frac{\alpha}{\alpha+\beta} \cdot\left(1-P_{d}\right) \cdot \frac{T_{P}-T s}{T_{P}}
$$

Where $T_{p}$ is the sensing period. Note that $P_{i}$ is a function of sensing time $T s$.

By substituting (11) and (13) into (12), we have the handoff delay as follows:

$$
\begin{aligned}
\min \mathrm{T}_{\text {delay }}= & {\left[P_{d} P\left(H_{1}\right)+P_{f} P\left(H_{0}\right)\right] } \\
& \cdot\left\{[ 1 - P ( H _ { 1 } ) ^ { N } ] \cdot \left[P\left(H_{0}\right) \cdot T s+P\left(H_{1}\right) P\left(H_{0}\right) \cdot 2 T s\right.\right. \\
& \left.\left.+\ldots+P\left(H_{1}\right)^{N-1} P\left(H_{0}\right) \cdot(N-1) T s\right]+\left[P\left(H_{1}\right)^{N} \cdot(N-1) T s\right]\right\} \\
\text { s.t. } \quad \mathrm{P}_{\mathrm{i}}= & P\left(H_{1}\right) \cdot\left(1-P_{d}\right) \cdot \frac{T_{P}-\tau}{T_{P}}<\zeta \\
\mathrm{P}_{\mathrm{d}}= & P_{d}^{*}, P_{f} \leq P_{f}^{*}
\end{aligned}
$$

\section{Simulation Results and Analysis}

In this section, our major objective is to demonstrate the handoff delay under interference constraints in the proposed spectrum handoff scheme. We use MATLAB software to simulate a multiple channels scenario. The high-priority PUs can interrupt the transmission of low-priority SUs. Furthermore, we assume that all PUs and SUs 
have the exponentially distributed packet lengths in our simulations. If not specified, the parameters are chosen as follows: $\mathrm{P}_{\mathrm{d}}{ }^{*}=0.9, \mathrm{P}_{\mathrm{f}}{ }^{*}=0.1, \mathrm{~T}_{\mathrm{p}}=100 \mathrm{~ms}, \gamma=-15 \mathrm{~dB}, \zeta=0.1$.

To better illustrate the impact of PU's arrival time on the delay performance of proposed handoff scheme, we plot the average handoff delay versus $P\left(H_{0}\right)$ from 0.1 to 1 in Figure 2. As can be seen, when the PU is idle for more and more times, the probability of idle channel $P\left(H_{0}\right)$ increases, the handoff delay decreases. That is because the SU has more chance to occupy the channel, the possibility of release and handoff channel decreases.

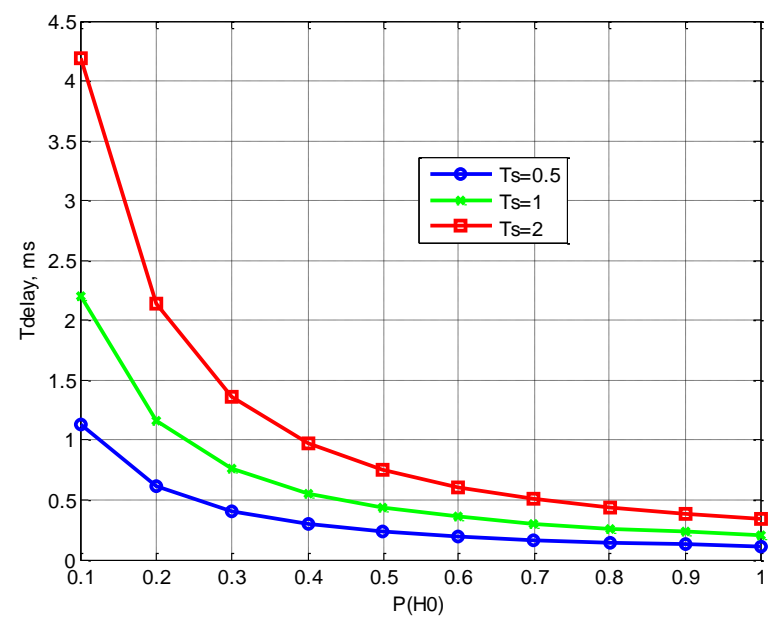
Figure 2. The Relationship between Handoff Delay $T_{\text {delay }}$ and $P\left(H_{0}\right)$ for
Different Sensing Time

We study the impact of interference on the handoff delay as in Figure 3, where interference probability $P_{i}$ increases from 0.02 to 0.08 for the case of $\mathrm{N}=5, \mathrm{~N}=10$ and $\mathrm{N}=15$, respectively. It is easy to find out the handoff delay increases with a larger interference probability. It can be explained that with larger interference probability, SUs could maintain its link on the channel with more chances before spectrum handoff. Therefore, we could set the lower interference constrains for the spectrum handoff scheme to protect the PUs.

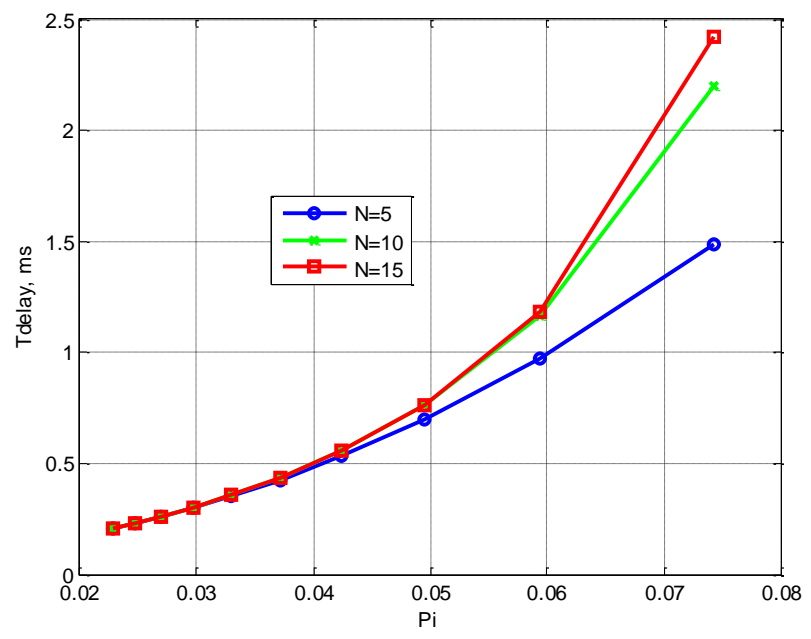

Figure 3. The Relationship between Handoff Delay $T_{\text {delay }}$ and Interference Probability Pi with Different Number of Channels 
Besides, we also interested in the impact of the number of channels $\mathrm{N}$ and sensing time $T s$ in the proposed spectrum handoff scheme. From Figure 4, we can infer that handoff delay increases with the increasing number of channels. That means the more the channels, the more chances for the SU's sensing, therefore, the more handoff delay.

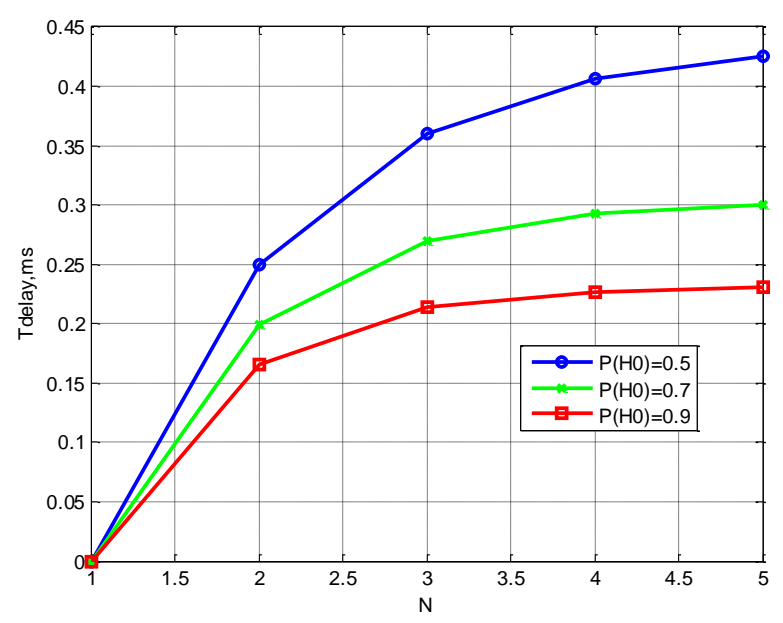

\section{Figure 4. The Relationship between Handoff Delay $T_{\text {delay }}$ and the Number of Channels $\mathbf{N}$ with Different $\mathrm{P}(\mathrm{H} 0)$}

Due to different spectrum sensing schemes, the sensing performance also impact the handoff delay. From Figure 5, we can see that the more sensing time, the larger handoff delay. That is because the SU performs the spectrum sensing to find the idle channel, the worse sensing performance, the more adverse effects on the spectrum handoff performance.

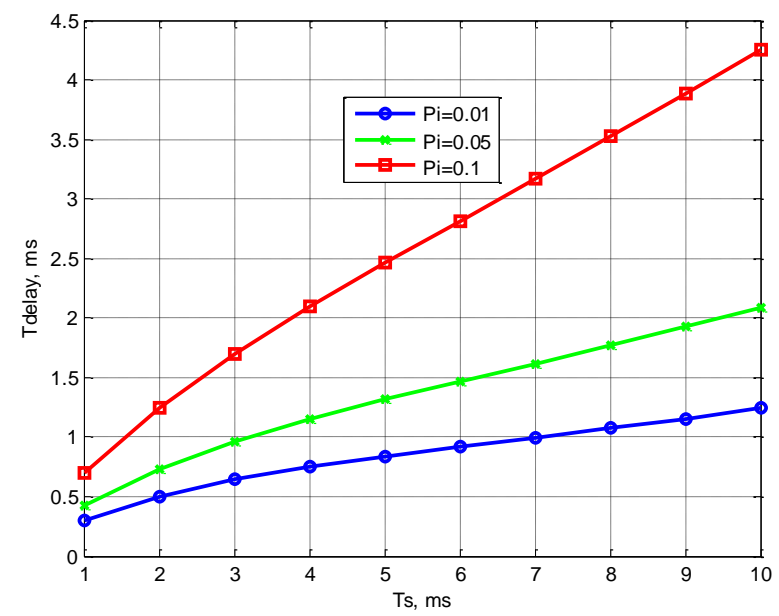

Figure 5. The Relationship between Handoff Delay $T_{\text {delay }}$ and Sensing Time Ts with Different Interference Constraint

\section{Conclusion}

In this paper, we present a spectrum handoff approach under the interference constraints in a multiple channels scenario. Spectrum handoff procedure is thoroughly investigated and handoff delay is derived in detail. Simulation results show that the 
proposed spectrum handoff scheme can not only protect the PUs, but also ensure the QoS provision of SUs in spectrum handoff scheme. Results indicate that the handoff delay is affected by factors, such as sensing time, the number of idle channel and the interference constrains and so on. Lower interference constrains can not only protect the PUS, but also can provide the lower handoff delay for SUs.

\section{Acknowledgements}

This work was supported by Scientific Research Fund of Heilongjiang Provincial Education Department (No. 12541583).

\section{References}

[1] S. Haykin, "Cognitive radio: brain-empowered wireless communications", IEEE Journal on Selected Areas in Communications, vol. 23, no. 2, (2005), pp. 201-220.

[2] Y. Song and X. Jiang, "ProSpect: A Proactive Spectrum Handoff Framework for Cognitive Radio Ad Hoc Networks without Common Control Channel”, IEEE Transactions on Mobile Computing, vol. 11, no. 7, (2012), pp. 1127-1139.

[3] S. D. Nguyen, T. L. Pham and D. S. Kim, "Dynamic spectrum handoff for industrial cognitive wireless sensor networks", Proceedings of the 11th IEEE International Conference on Industrial Informatics (INDIN), (2013), pp. 92-97, Bochum, Germany.

[4] E. Trigui, M. Esseghir and L. M. Boulahia, "Spectrum handoff algorithm for mobile cognitive radio users based on agents' negotiation", Proceedings of IEEE 9th International Conference on Wireless and Mobile Computing, Networking and Communications (WiMob), (2013), October 7-9, Lyon, pp. $750-756$.

[5] A. Bicen, E. Pehlivanoglu and S. Galmes, "Dedicated Radio Utilization for Spectrum Handoff and Efficiency in Cognitive Radio Networks", IEEE Transactions on Wireless Communications, vol. PP no. 99, (2013), pp. 1-9.

[6] W. Chengyu, H. Chen and J. Lingge," Spectrum handoff scheme based on recommended channel sensing sequence.China Communications", vol. 10, no. 8, (2013), pp. 18-26.

[7] C.W. Wang and L.C. Wang, "Analysis of Reactive Spectrum Handoff in Cognitive Radio Networks", IEEE Journal on Selected Areas in Communications, vol.30, no. 10, (2012), pp. 2016-2028.

[8] P.S., M.W. Rocha da Silva and J.F. de Rezende, "Detection error aware spectrum handoff mechanism for cognitive radios", Proceedings of 7th International ICST Conference on Cognitive Radio Oriented Wireless Networks and Communications (CROWNCOM), (2012), pp. 48-53, Stockholm, Sweden.

[9] S. Geirhofer, L. Tong and B. Sadler, "Cognitive Medium Access: Constraining Interference Based On Experimental Models", IEEE Journal on Selected Areas in Communications, vol. 26, no. 1, (2008), pp. 1-10.

[10] IEEE 802.22 Wireless RAN. Functional requirements for the 802-22WRAN standard, IEEE 802.2205/0007r46", (2005).

[11] Sahai and D. Cabric, "Spectrum sensing: fundamental limits and practical challenges", Proceedings of IEEE International Symposium on New Frontiers in Dynamic Spectrum Access Networks (DySPAN), (2005), Baltimore, MD.

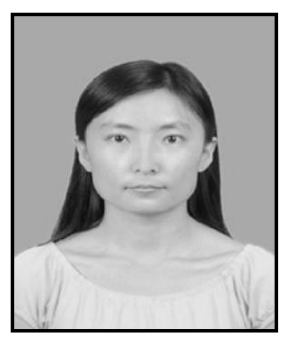

Hong Du, She was born in Heilongjiang Province, China, in 1982. She received the B.S. degree from the Northeast Petroleum University, Daqing, in 2006 and the M.S. degree from the Northeast Petroleum University, Daqing, in 2009, both in communication engineering. She received the Ph.D. degree from Beijing University of Posts and Telecommunications (BUPT), Beijing, in 2012. She is currently working in Heilongjiang Bayi Agriculture University. Her research interests include cognitive radio and internet of things. 

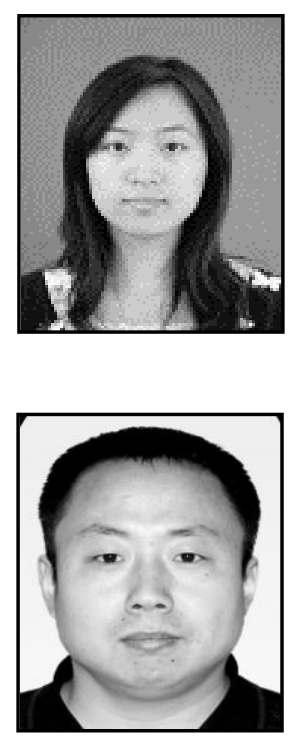

Shuang Fu, She was born in Heilongiiang Province, China, in 1982. She received the B.S. degree from the Heilongjiang University, Haerbin, in 2004 and the M.S. degree from the Northeast Petroleum University, Daqing, in 2010, both in communication engineering. She received the Ph.D. degree from Haerbin Engineering University (HEU), Haerbin, in 2014. She is currently working in Heilongjiang Bayi Agriculture University. Her research interests include cognitive radio.

Tingyi Shang, He was born in Shandong Province, China, in 1981. He received the B.S. degree from the Qufu Normal University, Qufu, in 2003 and the M.S. degree from the Zhengzhou University, Zhengzhou, in 2006. He is currently studying Ph.D. degree from Harbin University of Science And Technology, Haerbin. He is currently working in Heilongjiang Bayi Agriculture University. His research interests include wireless communication. 